\title{
Covid-19 tweeting in English: Gender differences
}

\author{
Mike Thelwall; Saheeda Thelwall
}

How to cite this article:

Thelwall, Mike; Thelwall, Saheeda (2020). "Covid-19 tweeting in English: Gender differences". El profesional de la información, v. 29, n. 3, e290301.

https://doi.org/10.3145/epi.2020.may.01

Manuscript received on April 17th 2020 Accepted on April 30th 2020

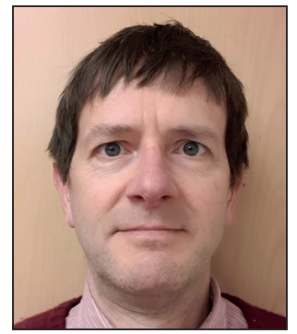

\author{
Mike Thelwall $\bowtie$ \\ https://orcid.org/0000-0001-6065-205X \\ University of Wolverhampton \\ Statistical Cybermetrics Research Group \\ Wulfruna Street \\ Wolverhampton WV1 1LY, UK \\ m.thelwall@wlv.ac.uk
}

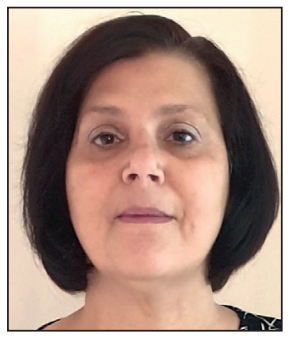

Saheeda Thelwall

https://orcid.org/0000-0002-0333-399X

Institute of Health, Faculty of Education Health \& Wellbeing

University of Wolverhampton

Wulfruna Street

Wolverhampton WV1 1LY, UK

s.thelwall@wlv.ac.uk

\begin{abstract}
At the start of 2020, Covid-19 became the most urgent threat to global public health. Uniquely in recent times, governments have imposed partly voluntary, partly compulsory restrictions on the population to slow the spread of the virus. In this context, public attitudes and behaviors are vitally important for reducing the death rate. Analyzing tweets about the disease may therefore give insights into public reactions that may help guide public information campaigns. This article analyses 3,038,026 English tweets about Covid-19 from March 10 to 23, 2020. It focuses on one relevant aspect of public reaction: gender differences. The results show that females are more likely to tweet about the virus in the context of family, social distancing and healthcare, whereas males are more likely to tweet about sports cancellations, the global spread of the virus, and political reactions. Thus, women seem to be taking a disproportionate share of the responsibility for directly keeping the population safe. The detailed results may be useful to inform public information announcements and to help understand the spread of the virus. For example, failure to impose a sporting bans whilst encouraging social distancing may send mixed messages to males.
\end{abstract}

\section{Keywords}

Covid-19; Coronavirus; Twitter; Gender; Public health information.

\section{Introduction}

Covid-19 is, at the time of writing, a major global threat to public health (e.g., Lipsitch; Swerdlow; Finelli, 2020). Public actions are critically important in slowing the spread of the virus and therefore reducing the death rate due to the volume of critically ill patients needing simultaneous care, given a limited supply of ventilators. Governments around the world have reacted by announcing mandatory actions, such as shutting restaurants and the normal functioning of schools, and by giving strongly recommended or mandatory advice to the public for personal hygiene and social distancing to slow the spread of the virus. The extent to which the population follows expert health advice is expected to have a substantial impact on the death rate from the virus. If social distancing is widely ignored or misunderstood, for example, then national healthcare facilities will not be able to give all critically ill patients the care that they need to survive. It is therefore vitally important to assess how the public is reacting to the crisis and one way (amongst many) of investigating this is through social media posts, including tweets (e.g., Cinelli; Quattrociocchi; Galeazzi et al., 2020), and one important potential arena of difference (amongst many, such as disability: Thelwall \& Levitt, 2020; life satisfaction: Li; Wang; Xue; Zhao; Zhu, 2020) is gender since women have traditionally taken more caring responsibilities (Carers UK, 2020).

Twitter has the advantage for research that it is typically fully public and researchers can therefore access its contents

Acknowledgements

Thank you to Dr Stephanie Haustein for comments on an earlier version of this paper. 
Twitter is a natural platform for public information sharing in many countries, including all large English-speaking nations. Although less popular than Facebook, its advantage for research is that it is typically fully public and researchers can therefore access its contents. Moreover, Twitter gives free use of an Applications Programming Interface (API) for automatically harvesting recent (up to a week old) tweets matching keyword searches, making it a practical source of data about public reactions to events. A disadvantage is that Twitter user demographics do not match those of the population. In the USA, about $23 \%$ of adults use the site, behind Facebook (71\%) and Instagram (38\%), but ahead of WhatsApp (18\%) and Reddit (13\%) (Schaeffer, 2019). Moreover, older people (and more at risk from Covid-19) are less likely to use Twitter, men are slightly more likely to use it (50\% of its US users are female but $52 \%$ of the US population are female) but adopters tend to be richer and more educated in the USA (Smith; Wojcik, 2019). There are also finer-grained differences, such as political variations between users and non-users (Smith; Hughes; Remy; Shah, 2020). Nevertheless, analyzing tweets may give some quick large-scale insights into public reactions to Covid-19.

This study focuses on gender differences in reactions to Covid-19 on Twitter. Since public safety measures must be adhered to by the entire population to be maximally effective and (majority female) careers have a particularly important role, any gender differences in responses may point to weaknesses in public communications about the seriousness of the outbreak. Gender differences have previously been found for the effectiveness of health-related behavioral change interventions, for example (e.g., Leblanc; Bégin; Hudon; Royer; Corneau; Dodin; Lemieux, 2014; Reinhardt; Bischof; Grothues; John; Meyer; Rumpf, 2008), and there were gender differences in safety behaviors (e.g., social distancing, hand washing) in China when affected by SARS (Lau; Yang; Tsui; Pang, 2004). This information may help with the creation of new messages targeting males or females more effectively. In addition, understanding gender differences may help modelling epidemiologists to create more accurate models of the spread of the disease. The current paper therefore analyses two weeks (March 10-23, 2020) of tweeting in English about Covid-19 from the perspective of gender differences in responses. Although the virus is a global pandemic, the focus on English is for pragmatic methodology reasons and similar research in other languages is encouraged (and supported by the free software at http://mozdeh.wlv.ac.uk).

\section{Methods}

The research design was to collect English-language tweets matching a set of queries related to Covid-19 over two weeks and to identify words used more by males than females and vice versa, using these to point to aspects of gender difference in tweeting about the virus. A word frequency method is useful for gender comparisons because it gives statistically significant evidence in a transparent fashion (Thelwall; Stuart, 2019). In contrast, content analysis or thematic analysis are unlikely to discover fine-grained gender differences and cluster-based methods, such as topic modelling, can be changed by small alterations in the data, and so are not robust. Topic modelling is also not able to give as fine-grained gender difference information as word frequency comparisons. Word frequency analysis therefore fills a gap in comparison to other methods.

The following queries were used to identify different common ways of referring to the disease: coronavirus; "corona virus"; Covid-19; Covid19. These were submitted to Twitter at the maximum speed allowed by the free Twitter API from 10 to 23 March 2020, obtaining 3,038,026 tweets after eliminating duplicates (including multiple retweets) and near duplicates (tweets identical apart from @usernames and \#hashtags). The tweets were collected and analyzed with the free software Mozdeh:

http://mozdeh.wlv.ac.uk

This method will probably have matched a small minority of related tweets since there were country-specific hashtags, behavior-specific hashtags (e.g., \#socialdistancing) and most pandemic-related tweets probably did not explicitly mention its name. Nevertheless, the queries were effective at generating a large sample of relevant tweets.

Twitter does not record user genders, but it is possible to guess male and female genders (only) from their display name if it starts with a first name. A list of gendered first names was used to match the first part of Twitter display names. This list was US-based, since the USA is the major English-language user of Twitter and its population has international ethnic origins, so its names probably reflect to some extent the names in other anglophone countries. The list was derived from the 1990 US census (top 10,000 names) and supplemented by GenderApi.com (names with at least 100 US records). Names were included as female (respectively, male) from either source if at least $90 \%$ of people with the name were female (respectively, male). Twitter names (display names, rather than usernames) were split at the first space or non-alphanumeric character, first digit, or first camel case transition from lowercase to uppercase (e.g., MikeThelwall). The $90 \%$ threshold was chosen to give a high degree of certainty that the user was the given gender. The method is imperfect because Twitter usernames may be informal or not reflect a person's name (e.g., CricketFan938624), or based on a relatively gender-neutral name (e.g., Sam, Pat) or a rare name, including names from small ethnic minorities in the USA. Nevertheless, the first name procedure splits a set of tweets into three groups: (a) likely to be male-authored (24\%); (b) likely to be female-authored (16\%); (c) unknown (60\%). Comparing words in sets (a) and (b) gives an indication of likely 
gender differences overall. Visual inspection of the most active users in the data suggests that most bot and corporate tweets are assigned to the unknown gender set. There are more accurate alternative gender detection methods, such as by combining facial recognition and culture detection from last names (Karimi; Wagner; Lemmerich; Jadidi; Strohmaier, 2016), but this information is rarely available on Twitter.

Gender differences in topics were identified by a word frequency comparison method to identify words more used by either males or females. Words were used rather than phrases or lemmas extracted by natural language processing because informal language and hashtags are used on Twitter, which are problematic for linguistic processing. Natural language processing may also not work well on Covid-19 related tweets because of the technical terms and phrases associated with it.

For each word, the proportion of female-authored tweets containing the word was compared to the proportion of male-authored tweets containing the word using a $2 \times 2$ chi-square test for the table:

[[Female tweets with word, Female tweets without word],[Male tweets with word, Male tweets without word]].

A statistically significant chi-squared value (3.841 for $p=0.05)$ gives evidence to reject the null hypothesis of no gender difference in use of the word. Because the test is repeated for every word and there are 1,372,497 words, this procedure would almost certainly produce tens of thousands of false positives due to the number of tests. The Benjamini-Hochberg procedure (Benjamini; Hochberg, 1995) was used to correct for this. It is a familywise error rate correction method that ensures that the probability of incorrectly rejecting the null hypothesis in any test is below a threshold value. For extra power, words that were too rare to trigger a statistically significant result, even they were only used by males (or females) were not tested. This chisquared/Benjamini-Hochberg approach for detecting gender differences in term frequencies has previously been used for academic abstracts (Thelwall; Bailey; Makita; Sud; Madalli, 2019; Thelwall; Bailey; Tobin; Bradshaw, 2019), Reddit posts (Thelwall; Stuart, 2019) and YouTube comments (Thelwall, 2018). The procedure was repeated three times, for $p=0.05, p=0.01$, and $p=0.001$, recording the highest significance level for each word.

The above procedure was also applied to each day separately to determine the statistically significantly gendered terms for each day (i.e., 14 additional sets of tests). This extra step was taken because a word that is gendered on a single day seems likely to be less relevant to Covid-19 than a word that is gendered on multiple days. For example, a one-day gendered term might relate to a news event that was affected by Covid-19 (e.g., a sporting event cancellation) but this might not be important to the ongoing discussion of the virus. The threshold for including a term was set at (the equivalent of) more than two highly statistically significant days. Allocating one star to significance at $p=0.05$, two for $p=0.01$ and three for $p=0.001$, the threshold requirement was a total of at least seven stars over the fourteen days. This threshold gave a total of 102 terms out of the 339 that were statistically significantly gendered on at least one day. A single combined list was used rather than a set of daily lists to focus on longer-term issues.

Each word judged statistically significantly gendered (either overall, or on multiple days) reflects one or more underlying gender differences in motivations for tweeting or a gender difference in language styles. Each term's underlying causes can be inferred by reading a random sample of tweets containing the term, known as the Key Word In Context (KWIC) method (Luhn, 1960). For example, the term league was associated with tweets discussing the full or partial closure of various sporting competitions or facilities. Gender differences in this word therefore suggest that males were more likely to tweet that league-based sport was affected by Covid-19 restrictions. The word contexts varied from obvious (e.g., \#jantacurfew) to obscure (e.g., it). In particular, many pronouns were female associated, reflecting a people-focus rather than a topic, and definite and indefinite articles were male-associated, reflecting an information focus rather than a specific topic. In cases where the context of a term was unclear from reading ten randomly selected tweets (using the random sort option in Mozdeh), a word association analysis was run on the term to identify top associating terms to give additional insights into its main use contexts. This word association analysis (a button in Mozdeh) lists words that co-occur disproportionately often with the selected term to identify its core associations. For example, the terms that co-occur disproportionately often with "hand" include washing, sanitizer and shake, suggesting that hands are discussed mostly in the context of the need to wash them but not shake them.

The words were manually grouped into themes by the first author only (for a quick reaction analysis, but the dataset is available online to check) for each gender to highlight the main types of gender difference.

\section{Results}

The main themes identified in the tweets are summarized below by gender. The complete list of terms and raw tweet counts associated with them are available on FigShare (Thelwall, 2020).

\subsection{Male-oriented themes}

Male-authored tweets about Covid-19 were about twice as likely as females to discuss sports, typically in the context of speculation about, or announcements of, events or competitions being cancelled (Figure 1). Whilst this is relatively peripheral to the disease, males were also substantially more likely to mention, or take issue with, political figures or government, particularly within India (Figure 2). Males were also more likely to tweet about the economy (terms: economy, 
market; not graphed). The near absence of the UK and USA from the geographic terms (exception: Liverpool), despite the focus on English might reflect weaker gender differences in these countries.

The epidemiology of the virus (Figure 3), including its geographic spread (Figure 4), was another male topic. Both relate to sharing news about the spread and extent of the virus.

\subsection{Female-oriented themes}

Female-oriented themes seemed to focus on the first and second lines of defense against the virus. The key theme of social distancing is moderately female-oriented (Figure 5), in the sense that females were more likely to use the \#socialdistancing hashtag and the need to stay at home as far as possible. Partly related to social distancing but also to lockdowns, females were more likely to mention family members (Figure 6) and to use all pronouns (Figure 7). Pronouns were typically used for a mix of purposes but tweets with pronouns or family members seemed more likely to discuss concrete actions or practical implications for the tweeter and the people that they know. Thus, all three themes have a practical and personal orientation. Females were also more likely to tweet about education (terms: school, student, teacher; not graphed), presumably due to its impact on themselves or their family.

Females were also more likely to discuss healthcare issues (Figure 8). These tweets were less focused on immediate practical issues but on the main line of defense against the virus, should the practical steps fail. Related to this, females were also more likely to express gratitude to healthcare workers and others (terms not graphed) and anxiety (see below).

\subsection{Mixed gender}

Two broad themes were mixed gender in the sense of some terms being male-associated and others being female-associated. Males were more likely to discuss the virus as a war whereas females were more likely to mention their anxiety about its effects (Fig. 9). The war metaphor (e.g., the fight against (ovid) is a way of generalizing the situation as well as perhaps for males glamorizing actions against it, or emphasizing the seriousness of the issue. Thus, war metaphors could be an indirect way of expressing anxiety.

There were mixed gender differences in discussions of curfews (Figure 10). Whilst

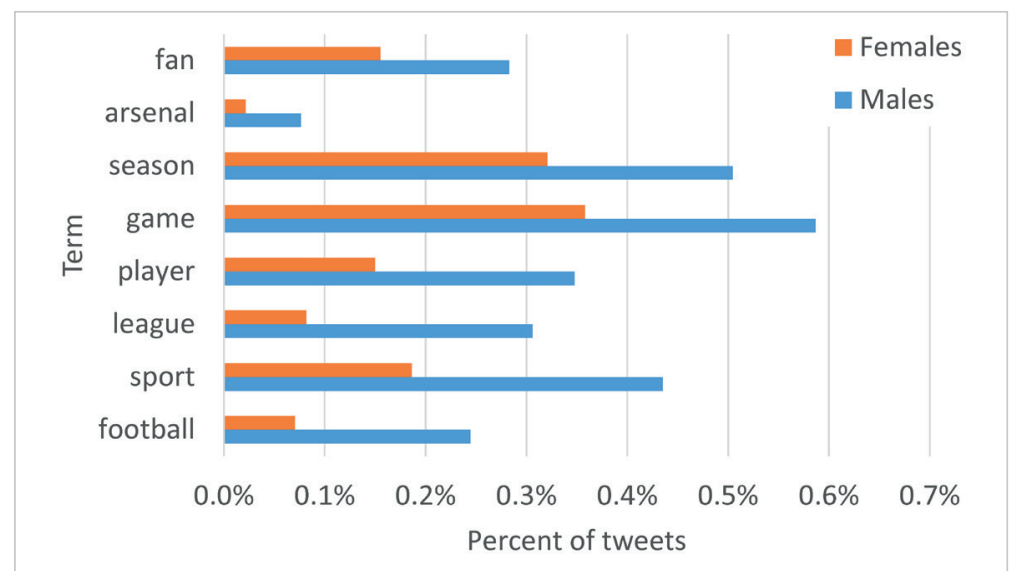

Figure 1. Sport-related terms with statistically significant gender differences in usage.

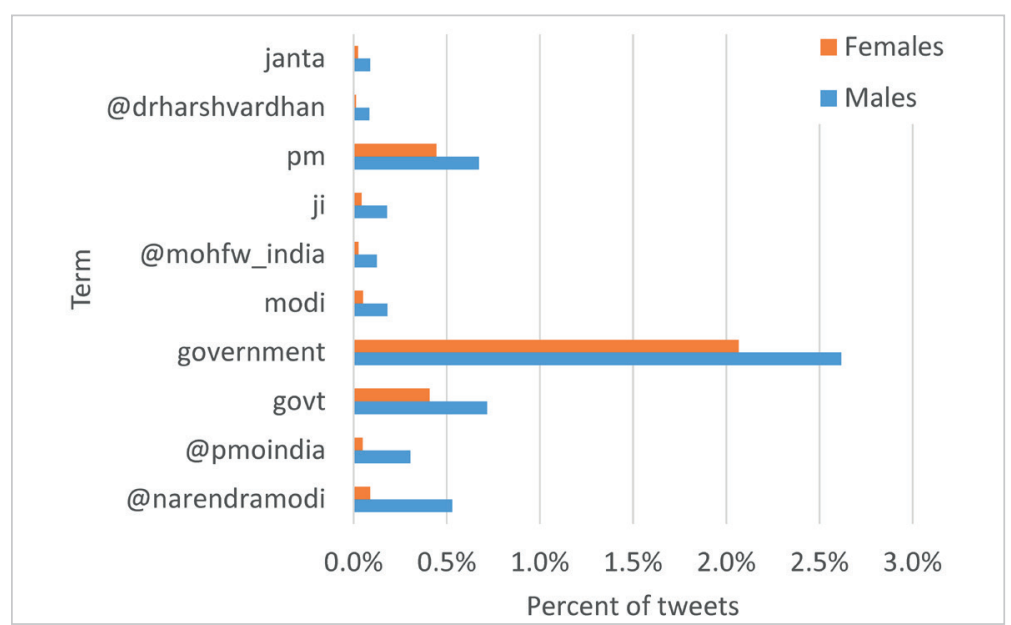

Figure 2. Politics-related terms with statistically significant gender differences in usage.

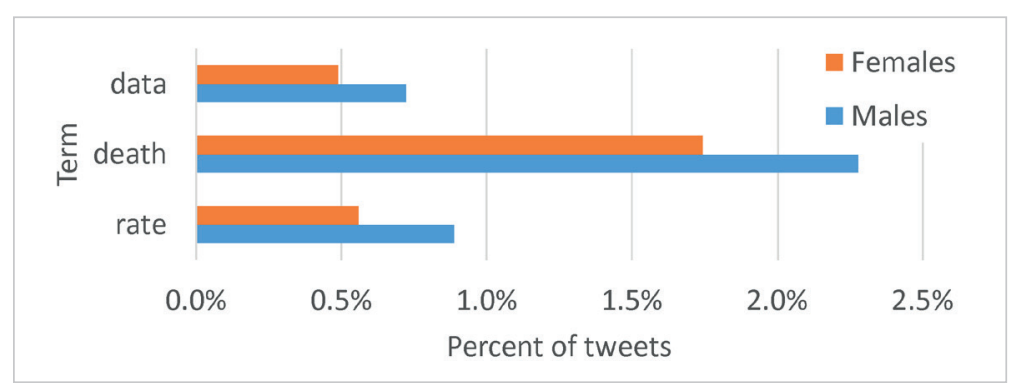

Figure 3. Epidemiology-related terms with statistically significant gender differences in usage.

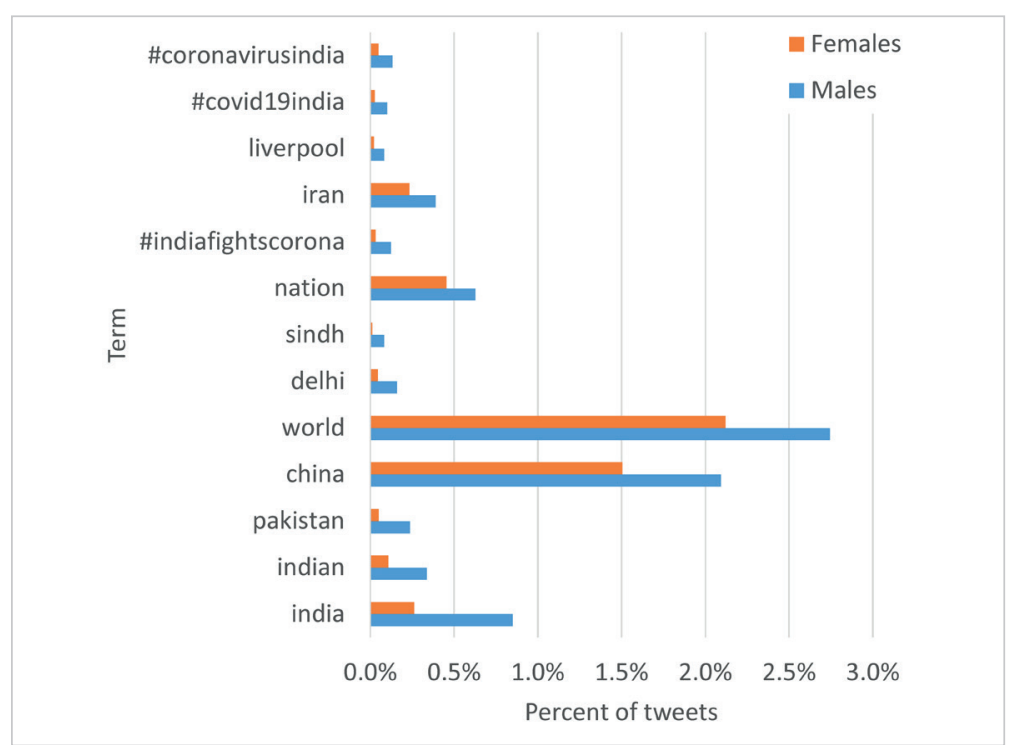

Figure 4. Geography-related terms with statistically significant gender differences in usage (Liverpool is also sport-related). 
males were more likely to announce the existence of a curfew, females were more likely to discuss its practical impacts.

\section{Discussion}

This quick analysis of gender differences in English tweeting about Covid-19 has several limitations. In addition to the issues discussed above, another important aspect is that Twitter does not report the geographic location of the tweets and so the data has unknown origins. Whilst some geographic origins can be identified from tweets and user self-descriptions (Kulshrestha; Kooti; Nikravesh; Gummadi, 2012), this is a slow process and not appropriate here. In particular, if some countries have an unusually high proportion of active tweeters of one gender, then this could translate into tweets about that country statistically significantly associating with that gender with the tests used above. Another limitation is that the data concerns tweet authors but not tweet readers, and is it possible, for example, that males read tweets about families (or think about them) as much as females but tweet less on the topic.

The results are broadly consistent with previous research into gender differences in language use, and gender differences in interests, such as sport and politics, as well as nursing as a career. The greater male interest in sport in many countries is widely known (e.g., Plaza; Boiché; Brunel; Ruchaud, 2017), and males also seem to discuss politics more (or at least more directly: Bode, 2017). Presumably the sport gender differences found would tend to decrease during the pandemic, however, due to the cancellation of sporting events. The greater female focus on caring (65\% of family caregivers are female in the USA: $\mathrm{Fa}$ mily Caregiver Alliance, 2019), and family (Parker; Horowitz; Rohal, 2015) has also been found before. In terms of language use, females have often been found to use personal pronouns more in some types of text (Argamon; Koppel; Fine; Shimoni, 2003), including on social media (ThelwaII, 2018). The primary contribution of our contribution is therefore to so show which gender differences translate to Covid-19 on Twitter, rather than finding new gender differences.

\section{Conclusions}

Although these conclusions are drawn from statistical tests on big data from Twitter, inferences from the results are tentative due to the processing limitations above that

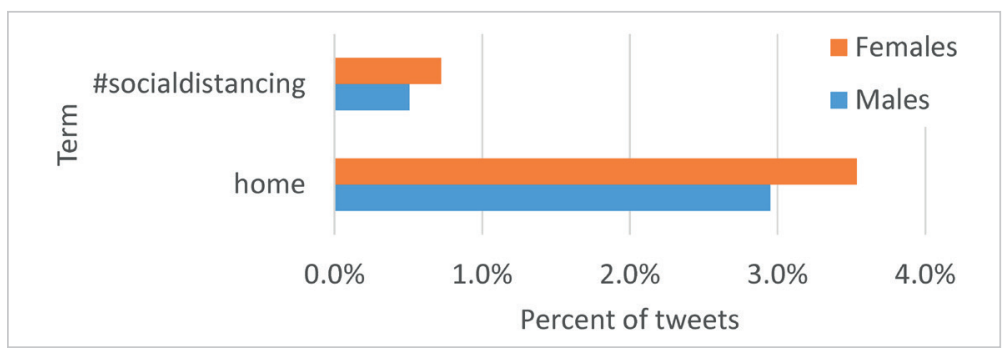

Figure 5. Social distancing-related terms with statistically significant gender differences in usage.

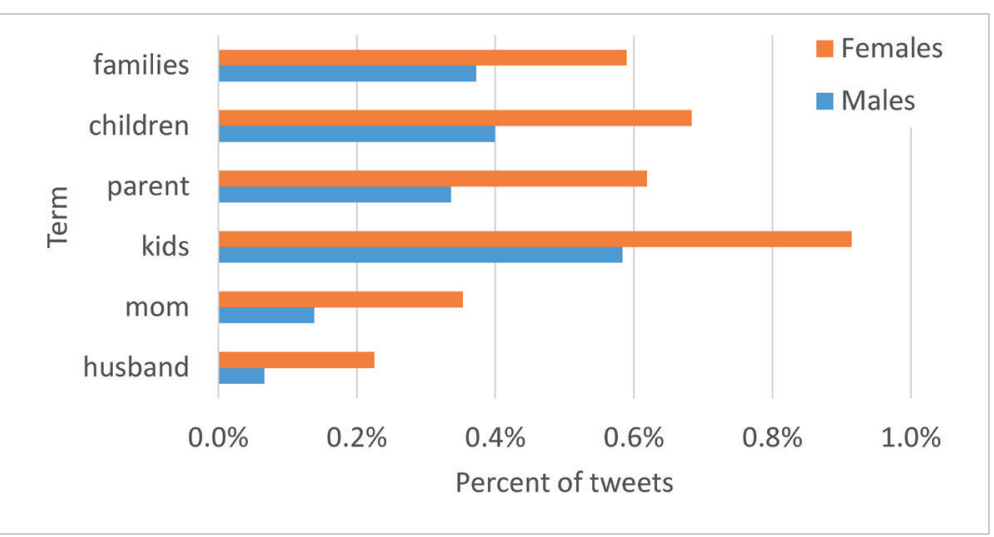

Figure 6. Family-related terms with statistically significant gender differences in usage.

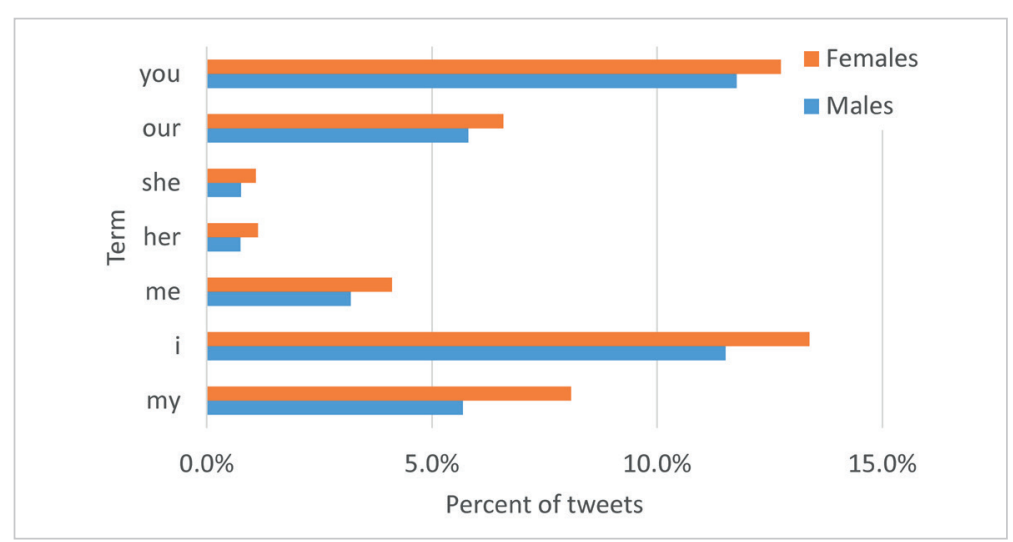

Figure 7. Pronouns with statistically significant gender differences in usage.

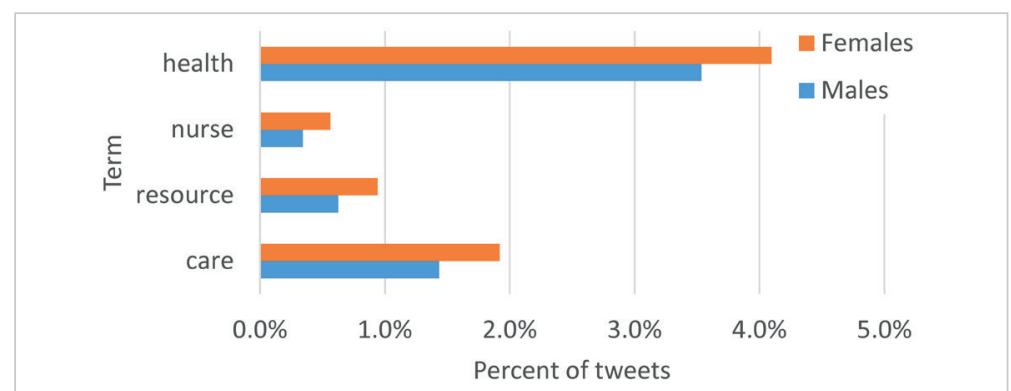

Figure 8. Healthcare-related terms with statistically significant gender differences in usage.

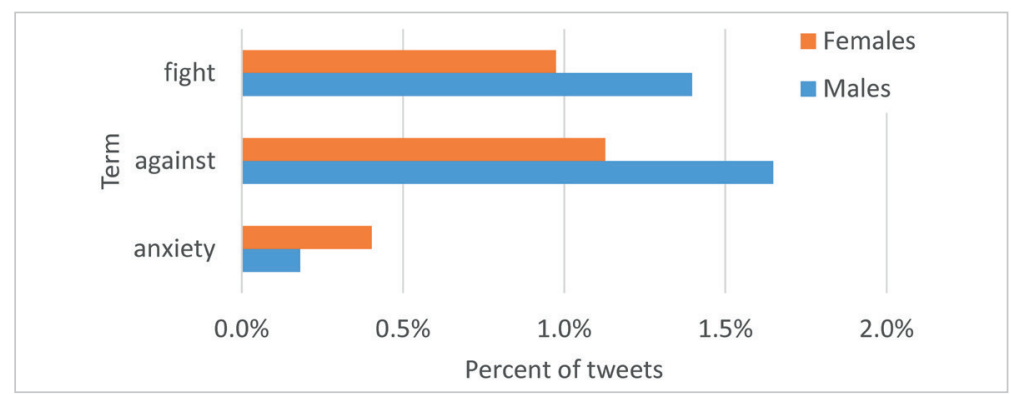

Figure 9. Fight or worry-related terms with statistically significant gender differences in usage. 
could not be addressed and the lack of evidence connecting offline actions to the content of tweets. Thus, for example, the greater female tendency to tweet about families does not prove that females were more concerned about the welfare of their families due to Covid-19, although this is a plausible explanation. Thus, the conclusions should be treated similarly to those of purely qualitative research: as evidence-based ideas but not proof of those ideas.

The substantially greater focus of males on sport in tweets about Covid-19 might be taken as evidence that males were less serious about the disease in the initial sta-

ges. Irrespective of whether this is true, sport was an important factor in the reaction to Covid-19 for many males. A policy-related suggestion from this is that cancelling sporting events may be particularly effective in communicating to males the seriousness of a situation. For example, if the population is told to socially distance but allowed to attend mass sporting events on the basis that an alternative (watching the event in crowded pubs or bars) is more dangerous, this may send mixed messages since crowded sporting events clearly involve close proximity with large numbers of strangers. Thus, any relaxation of bans on sporting events should be considered very carefully in the future, in countries where they are in place, and sporting bans should be considered in other countries as an important component of social distancing strategies, both for the spreading risk and the message sent to (mainly) males.

The results are consistent with, but do not prove, that women are at the forefront of actions to prevent the spread of Covid-19. Public health officials, whilst targeting everyone, might therefore need to be particularly careful that core messages are transmitted effectively to women in media that they consume so that social distancing is fully understood by as many as possible so that it can be carried out as effectively as possible.

\section{References}

Argamon, Shlomo; Koppel, Moshe; Fine, Jonathan; Shimoni, Anat-Rachel (2003). "Gender, genre, and writing style in formal written texts". Text, v. 23, n. 3, pp. 321-346.

https://www.researchgate.net/publication/2914978_Gender_Genre_and_Writing_Style_in_Formal_Written_Texts

Benjamini, Yoav; Hochberg, Yusuf (1995). "Controlling the false discovery rate: a practical and powerful approach to multiple testing". Journal of the Royal Statistical Society: Series B (Methodological), v. 57, n. 1, pp. 289-300. https://doi.org/10.1111/j.2517-6161.1995.tb02031.x

Bode, Leticia (2017). “Closing the gap: gender parity in political engagement on social media”. Information, communication \& society, v. 20, n. 4, pp. 587-603.

https://doi.org/10.1080/1369118X.2016.1202302

Carers UK (2020). 10 facts about women and caring in the UK on International women's day.

https://www.carersuk.org/news-and-campaigns/features/10-facts-about-women-and-caring-in-the-uk-on-internationalwomen-s-day

Cinelli, Matteo; Quattrociocchi, Walter; Galeazzi, Alessandro; Valensise, Carlo-Michele; Brugnoli, Emanuele; Schmidt, Ana-Lucia; Zola, Paola; Zollo, Fabiana; Scala, Antonio (2020). “The covid-19 social media infodemic". arXiv preprint arXiv:2003.05004. https://arxiv.org/abs/2003.05004

Family Caregiver Alliance (2020). Caregiver statistics: Demographics.

https://www.caregiver.org/caregiver-statistics-demographics

Karimi, Fariba; Wagner, Claudia; Lemmerich, Florian; Jadidi, Mohsen; Strohmaier, Markus (2016). "Inferring gender from names on the web: A comparative evaluation of gender detection methods". In: Proceedings of the $25^{\text {th }}$ International conference companion on World Wide Web (pp. 53-54).

https://arxiv.org/abs/1603.04322

Kulshrestha, Juhi; Kooti, Farshad; Nikravesh, Ashkan; Gummadi, Krishna P. (2012). "Geographic dissection of the Twitter network". In: Sixth international AAAl conference on weblogs and social media.

https://www.aaai.org/ocs/index.php/ICWSM/ICWSM12/paper/viewPaper/4685 
Lau, Joseph; Yang, Xilin; Tsui, Hiyi; Pang, Ellie (2004). "SARS related preventive and risk behaviours practised by Hong Kong-mainland China cross border travellers during the outbreak of the SARS epidemic in Hong Kong". Journal of epidemiology \& community health, v. 58, n. 12, pp. 988-996.

https://doi.org/10.1136/jech.2003.017483

Leblanc, Vicky; Bégin, Catherine; Hudon, Anne-Marie; Royer, Marie-Michelle; Corneau, Louise; Dodin, Sylvie; Lemieux, Simone (2014). "Gender differences in the long-term effects of a nutritional intervention program promoting the Mediterranean diet: changes in dietary intakes, eating behaviors, anthropometric and metabolic variables". Nutrition journal, v. 13, n. 1, article 107.

https://doi.org/10.1186/1475-2891-13-107

Li, Sijia; Wang, Yilin; Xue, Jia; Zhao, Nan; Zhu, Tingshao (2020). "The impact of Covid-19 epidemic declaration on psychological consequences: A study on active Weibo users". International journal of environmental research and public health, v. 17, n. 6, article 2032.

https://doi.org/10.3390/ijerph17062032

Lipsitch, Marc; Swerdlow, David; Finelli, Lyn (2020). "Defining the epidemiology of Covid-19 - studies needed". New England journal of medicine, n. 382, pp. 1194-1196.

https://doi.org/10.1056/NEJMp2002125

Luhn, Hans (1960). "Key word-in-context index for technical literature (kwic index)". American documentation, v. 11, n. 4, pp. 288-295.

Parker, Kim; Horowitz, Juliana; Rohal, Molly (2015). Parenting in America: Outlook, worries, aspirations are strongly linked to financial situation. Pew Research Center.

https://www.pewsocialtrends.org/wp-content/uploads/sites/3/2015/12/2015-12-17_parenting-in-america_FINAL.pdf

Plaza, Mélissa; Boiché, Julie; Brunel, Lionel; Ruchaud, François (2017). "Sport = male... But not all sports: Investigating the gender stereotypes of sport activities at the explicit and implicit levels". Sex roles, v. 76, n. 3-4, pp. 202-217.

https://doi.org/10.1007/s11199-016-0650-x

Reinhardt, Susa; Bischof, Gallus; Grothues, Janina; John, Ulrich; Meyer, Christian; Rumpf, Hans-Jürgen (2008). “Gender differences in the efficacy of brief interventions with a stepped care approach in general practice patients with alcohol-related disorders". Alcohol \& alcoholism, v. 43, n. 3, pp. 334-340.

https://doi.org/10.1093/alcalc/agn004

Schaeffer, Katherine (2019). U.S. has changed in key ways in the past decade, from tech use to demographics. Pew Research Center.

https://www.pewresearch.org/fact-tank/2019/12/20/key-ways-us-changed-in-past-decade

Smith, Aaron; Hughes, Adam; Remy, Emma; Shah, Sono (2020). Democrats on Twitter more liberal, less focused on compromise than those not on the platform. Pew Research Center.

https://www.pewresearch.org/fact-tank/2020/02/03/democrats-on-twitter-more-liberal-less-focused-on-compromisethan-those-not-on-the-platform

Smith, Adam; Wojcik, Stefan (2019). 10 facts about Americans and Twitter. Pew Research Center. https://www.pewresearch.org/fact-tank/2019/08/02/10-facts-about-americans-and-twitter

Thelwall, Mike; Bailey, Carol; Makita, Meiko; Sud, Pardeep; Madalli, Devika P. (2019). “Gender and research publishing in India: Uniformly high inequality?". Journal of informetrics, v. 13, n. 1, pp. 118-131.

https://doi.org/10.1016/j.joi.2018.12.003

Thelwall, Mike; Bailey, Carol; Tobin, Catherine; Bradshaw, Noel-Ann (2019). "Gender differences in research areas, methods and topics: Can people and thing orientations explain the results?". Journal of informetrics, v. 13, n. 1, pp. 149-169. https://doi.org/10.1016/j.joi.2018.12.002

Thelwall, Mike; Levitt, Jonathan (2020). "Retweeting Covid-19 disability issues: Risks, support and outrage". El profesional de la información, v. 29, n. 2, e290216.

https://doi.org/10.3145/epi.2020.mar.16

Thelwall, Mike; Stuart, Emma (2019). "She's Reddit: A source of statistically significant gendered interest information?". Information processing \& management, v. 56, n. 4, pp. 1543-1558.

https://doi.org/10.1016/j.ipm.2018.10.007

Thelwall, Mike (2018). “Can museums find male or female audiences online with YouTube?". Aslib journal of information management, v. 70, n. 5, pp. 481-497.

https://doi.org/10.1108/AJIM-06-2018-0146

Thelwall, Mike (2020). "Gender differences in Covid-19 tweeting in English". FigShare.

https://doi.org/10.6084/m9.figshare.12026625 